\title{
A Case Report: Cystic Clear Cell Tumor of Borderline Malignancy of the Ovary
}

\author{
Yuko Matsubara*, Toru Fujioka, Keiichi Matsubara, Akihiro Nawa \\ Department of Obstetrics and Gynecology, Ehime University School of Medicine, Toon, Japan \\ Email: ${ }^{*}$ takeyu@m.ehime-u.ac.jp
}

Received 4 April 2014; revised 2 May 2014; accepted 9 May 2014

Copyright (C) 2014 by authors and Scientific Research Publishing Inc.

This work is licensed under the Creative Commons Attribution International License (CC BY). http://creativecommons.org/licenses/by/4.0/

(c) () Op Och Access

\begin{abstract}
Introduction: Ovarian borderline clear cell tumor is a very rare tumor accounting for less than $1 \%$ of borderline ovarian tumors. Currently, only 30 cases of borderline clear cell tumors have been reported. This borderline ovarian clear cell tumor often arises from endometriosis. But there are no definitive guidelines for the management of endometriosis with malignant transformation due to a lack of understanding of the mechanistic details that mediate the transformation of endometriosis into ovarian cancer. Case: A 52-year-old woman (G2 P2) who periodically underwent ultraso-nography over the course of 12 years in order to follow the endometrioma present in her left ovary. The diameter of the lesion was $46 \mathrm{~mm}$ and it remained largely unchanged. However, a solid region within the ovarian cyst was detected. After the surgery, the final pathological diagnosis was cystic clear cell tumor of borderline malignancy. Conclusion: It reported that risk factors of malignant transformation are older age, postmenopausal status, and larger tumor diameters were associated with a higher prevalence of cancer development. But it is recommended that patients with endometriosis, regardless of patient age or tumor size, undergo careful monitoring to detect changes in growth or the formation of solid regions for as long as the lesion is present.
\end{abstract}

\section{Keywords}

Borderline Malignancy, Clear Cell Tumor, Ovary

\section{Introduction}

Most Ovarian clear cell carcinoma is diagnosed as an invasive carcinoma when it is newly found and it comprises $2 \%-3 \%$ of all epithelial ovarian neoplasm. Borderline ovarian clear cell tumor is a rare tumor accounting for less than $1 \%$ of borderline ovarian tumors. Currently, only 30 cases of borderline clear cell tumors have been

${ }^{*}$ Corresponding author. 
reported. Therefore, organized screening is needed to find it early. This borderline ovarian clear cell tumor often arises from endometriosis. But there are no definitive guidelines for the management of endometriosis with malignant transformation. It is reported that risk factors of malignant transformation are older age, postmenopausal status, and larger tumor diameter were associated with a higher prevalence of cancer development. They defined postmenopausal women with ovarian endometriosis of $9 \mathrm{~cm}$ or larger in diameter as a high risk group and recommended extensive therapy, including surgery [1]. This report demonstrates a case of borderline ovarian clear cell tumor arising from endometriosis, whose size was small and unchanged for 12 years.

\section{Case}

52-year-old woman (G2 P2) who periodically underwent ultrasonography over the course of 12 years in order to follow the endometrioma present in her left ovary. The diameter of the lesion was $46 \mathrm{~mm}$ and it remained largely unchanged. However, during a recent clinical examination, a solid region within the ovarian cyst was detected and the patient was referred to our hospital for further examination.

Magnetic resonance imaging (MRI) confirmed that the $46 \mathrm{~mm}$ cyst contained a solid region (Figure 1(a)) that did not undergo enhancement following administration of Gadrinium (Figure 1(b)). Similarly, no significant accumulation of fluorodeoxyglucose (FDG) was observed within the solid region with positron emission tomography-computed tomography (PET-CT) (Figure 2). Cervical and endometrial cytology examinations were also negative. Laboratory data were within normal ranges, including the tumor markers CA125 assayed.

Based on the preoperative diagnosis of a suspicious malignant ovarian tumor, a diagnostic laparotomy was performed. An intraoperative rapid diagnosis indicated clear cell carcinoma was present within the ovarian tumor. Moreover, neither dissemination nor metastasis was found in the peritoneal cavity. A total abdominal hysterectomy with bilateral salpingo-oophorectomy and pelvic and para-aortic lymphadenectomy was subsequently performed.

Histopathological examination demonstrated that hobnail cells are present, however they do not exhibit stromal invasion. The final pathological diagnosis was cystic clear cell tumor of borderline malignancy (Figure 3). The postoperative course for the patient was uneventful, and no signs of recurrence were observed for two years.

\section{Discussion}

Endometriosis is a common gynecological condition that affects $10 \%$ of women of childbearing age. It is a chronic disease that is characterized by the presence of ectopic endometrial tissue outside the uterus. Furthermore, this disease is typically associated with painful symptoms, dysmenorrhea, dyspareunia, pelvic pain, infertility, and malignant transformation [2]. Approximately 1.0\% of endometriosis cases have been reported to undergo transformation to malignant tumors [3]. Persistent oxidative stress as a result of endometriosis-dependent

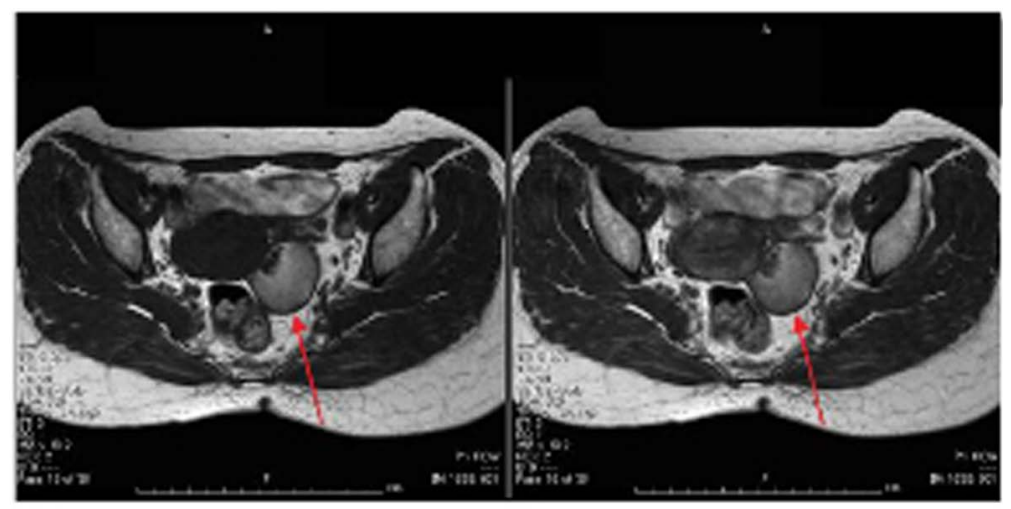

(a)

(b)

Figure 1. (a): Magnetic resonance imaging (MRI) scans (T1-weighted image) revealed the $46 \mathrm{~mm}$ cyst contained solid part; (b): Solid part was not enhanced after the administration of contrast material. 


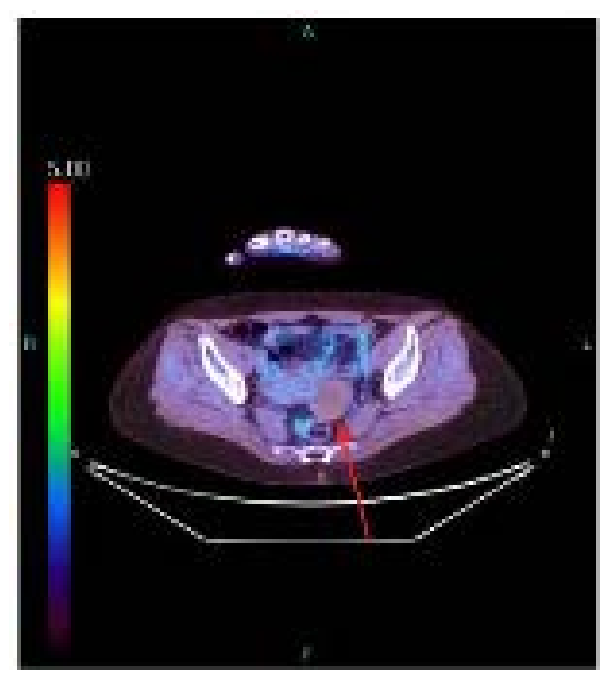

Figure 2. Positron emission tomography (PET$\mathrm{CT}$ ) revealed no significantly accumulation of FDG at the solid part.

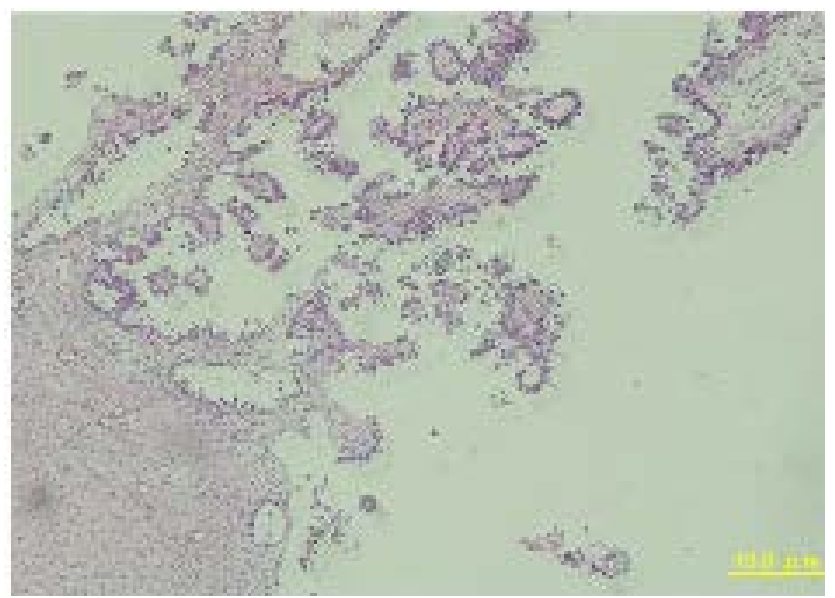

Figure 3. Histological finding of the ovarian tumor $(\times 100)$. Hobnail cells are present; however they do not exhibit stromal invasion.

hemorrhage is repeatedly associated with this malignant transformation. Such stressful microenvironment by inducing unique gene expression is called "Clear Cell Carcinoma Signature" [4].

Unlike serous and mucinous subtypes, most clear cell borderline ovarian tumors had occurred in the postmenopousal period. Uzan et al. reported [5] that peritoneal staging surgery is not required for the management of clear cell borderline ovarian tumors because all patients reported in the literature had stage I disease. They said clear cell borderline ovarian tumors carry a good prognosis. However, the power of this conclusion is limited.

There is no definite guideline for the management of endometriosis with special attention to malignant transformation, because of a lack of understanding of the natural history of these lesions and the risks of developing ovarian cancer. According to a Japanese cohort study, approximately $0.7 \%$ of patients with endometriosis subsequently developed ovarian cancer [3]. The authors further analyzed risk factors of malignant transformation and found that older age, postmenopausal status, and larger tumor diameter were associated with a higher prevalence of cancer development. They defined postmenopausal women with ovarian endometriosis of $9 \mathrm{~cm}$ or larger in diameter as a high risk group and recommended extensive therapy, including surgery [1].

In conclusion, a case of small cystic clear cell tumor of borderline malignancy that underwent transformation after 12 years is reported. It is reported that the prevalence of ovarian cancer was $0 \%$ among women with ova- 
rian endometriomas that were less than $6 \mathrm{~cm}$ in diameter [1]. But it is recommended that patients with endometriosis, regardless of patient age or tumor size, undergo careful monitoring to detect changes in growth or the formation of solid regions for as long as the lesion is present.

\section{Conflict of Interest}

The authors have no conflicts of interest in connection with submitted material.

\section{References}

[1] Kobayashi, H., Sumimoto, K., Kitanaka, T., et al. (2008) Ovarian Endometrioma-Risks Factors of Ovarian Cancer Development. European Journal of Obstetrics \& Gynecology and Reproductive Biology, 138, 187-193. http://dx.doi.org/10.1016/j.ejogrb.2007.06.017

[2] Rogers, P.A., D’Hooghe, T.M., Fazleabas, A., et al. (2009) Priorities for Endometriosis Research: Recommendations from an International Consensus Workshop. Reproductive Sciences, 16, 335-346. http://dx.doi.org/10.1177/1933719108330568

[3] Kobayashi, H., Sumimoto, K., Moniwa, N., et al. (2007) Risk of Developing Ovarian Cancer among Women with Ovarian Endometrioma: A Cohort Study in Shizuoka, Japan. International Journal of Gynecological Cancer, 17, 37-43. http://dx.doi.org/10.1111/j.1525-1438.2006.00754.x

[4] Mandai, M., Matsumura, N., Baba, T., et al. (2011) Ovarian Clear Cell Carcinoma as a Stress-Responsive Cancer: Influence of the Microenvironment on the Carcinogenesis and Cancer Phenotype. Cancer Letters, 310, 129-133. http://dx.doi.org/10.1016/j.canlet.2011.06.039

[5] Uzan, C., Dufeu-Lefebvre, M., Fauvet, R., et al. (2012) Management and Prognosis of Clear Cell Borderline Ovarian Tumor. International Journal of Gynecological Cancer, 22, 993-999. http://dx.doi.org/10.1097/IGC.0b013e3182534acf 